\title{
Proposal of the Coupled Thermomechanical Model of a Crank Mechanism
}

\author{
Bogumil CHILINSKI ${ }^{1}$ and Anna MACKOJC \\ Institute of Machine Design Fundamentals, Warsaw University of Technology, Poland
}

\begin{abstract}
The aim of the paper is to propose analytical coupled thermomechanical model of the crankshaft system, which includes the mutual interaction between thermodynamic and mechanical phenomena occurring in engines. The most relevant dynamic effects observable in the crank system are connected with its kinematics. When the mechanism operates there are also additional effects corresponding with stress, strain and thermal fields. Elastic properties of the system parts and changeable stiffness of the fuel-air mixture cause different dynamics of the entire device. The authors assumed that rigid motion of the crank mechanism, parts deformation and thermodynamic effects and their mutual dependencies will be included in the modelling process. Elasticity of the crankshaft system components is the reason for the difference between a rigid 'ideal' motion and the real movement of crankshaft elements. In most cases, it is enough to assume linear elastic material features based on the relatively high stiffness of the system preventing big deformations. This ensures small displacements and the correctness of the applied model. The performed investigations have shown an influence of the crank system flexibility on the overall device response. Moreover, the parameters that change due to thermodynamic and mechanical properties of the working medium were taken into account. The authors have applied simple engine cycles (Otto, Diesel or combined model) for determining engine load including the connection between mechanical and thermodynamic state variables. This caused another decrease of the total system stiffness. Further numerical testing proved a visible effect of the applied approach in the global system response. The main discrepancies are observable in natural frequencies and vibration modes. It can also be stated that the utilization of different engine cycles results in different engine features. The paper is concluded with an analysis of the existing systems and mutual reactions from the assumed phenomena. The authors have shown the necessity to take a transdisciplinary approach into account in order to model complex systems..
\end{abstract}

Keywords. Crank mechanism, dynamic model, engine vibration, coupled thermomechanical model, analytical modelling, Python and SymPy application

\section{Introduction}

Current trends in high efficiency engines and their low fuel consumption raise the need for the development of new concepts, application of extraordinary solutions and improvement of existing designs. This drives the demand for more sophisticated tools.

\footnotetext{
${ }^{1}$ Corresponding Author, Mail: bogumil.chilinski@pw.edu.pl.
} 
Models previously used become inaccurate and require a process of recreation that takes into account more physical phenomena in order to enable them to be closer to reality. In order to meet these requirements, a transdisciplinary approach in the modelling process $[3,8]$ shall be considered. Scientific papers regarding a merge of different scientific or industrial disciplines indicate the proposed new approach of investigation might relevantly enhance solving problems in design process methodology [11]. Moreover, they present the transdisciplinary modelling as an extensive discipline that might enable obtaining much more comprehensive results comparing with conventional approach. Furthermore, in the world-wide literature one might encountered scientific articles presenting the transdisciplinary engineering as unspecified in terms of having a precise or unique definition what allows to consider the paper's topic as transdisciplinary [10].

Operational problems of engines are connected mostly with dynamic phenomena occurring during operation. In most cases, they are caused by elastic and inertial properties of the engine components. The combination of both results in engine part vibrations affecting the system operability $[6,14]$. Moreover, a thermodynamic gas force which is the reason for limiting cycle existence impacts the system dynamics relevantly. In-depth analysis of the behaviour of system dynamics and a transdisciplinary approach can enable improved engine performance and its operational parameters.

Due to the complexity of the engine design, the designing process is based on a step-by-step improvement process, which leads to the final form of the particular motor. Designers are reluctant to introduce significant enhancements as that could lead to the unpredictable changes in dynamics of the motor. The satisfactory results of current engine designs are an effect of the confirmed industrial practice. However, due to power unit accessories design, more frequent process difficulties raise the need of new computational methods or models. Moreover, the required models should be accurate and fast. One of the projects related to engine equipment design was aimed on some efficient tools to be utilised in computations of Torsional Vibration Dampers [6]. The tools were required to be compatible with industrial specification and take into account manufacturing process of the object. The investigation carried out shows the necessity of transdisciplinary overcoming of limitations to ensure interchange of the older and current version of the part and dynamic conditions which lead to proper mechanical properties of the engine. For this purpose, a comprehensive engine model is necessary in order to allow precise system design process.

The main advantage of a transdisciplinary modelling are simultaneous considerations of mechanical and thermodynamic state variables. This allows for a complex analysis of the mutual influence of the system parameters on generated engine power. A typical approach assumes only the consideration of the mechanism geometry and dynamically independent changes of the rotational speed [1, 2]. Practical cases show there is an influence of the component vibrations on engine dynamics and operational parameters [7, 13]. These are mainly caused by additional stiffness of a fuel-air mixture or the crank system parts. This creates the need for a more detailed investigation of the engine complex model.

The engines main features are connected with the elastic properties of its components. Typically, they are not taken into account which limits the system vibrations [9, 12]. What is more, a crankshaft angle of rotation depends on the instantaneous state of the system. Even for a rigid crank and rod, the elasticity of the fuel-air mixture allows for small changes of the crank from its nominal position. 
Inclusion of the thermodynamic effect provides the nonlinear relationship of the gas force and state variables.

\section{Objectives and overview}

The aim of the paper is to propose the analytical coupled thermomechanical model of a crank mechanism, which considers mutual interactions between thermodynamic and mechanical phenomena occurring in engines. The proposed coupled model emphasises the importance of a transdisciplinary approach in modelling of complex systems. The authors proposed a mathematical model of the crank-piston mechanism in order to enable theoretical analysis and the concept identification. The model analysis is intended to determine the kinematics and dynamics of the crank system and to analyse the impact of the selected system parameters on the total system dynamic response.

Because of a preliminary nature of the investigation carried out, it considers only the analytical and the numerical investigation on the object. The authors states that it is necessary to recognise particular model features before Finite Element Analysis and further experimental investigation. Moreover, it was decided to take into account only the simplest models of the considered phenomena. This was motivated by the papers aim to propose a model with the ability to represent the coupling and the mutual relationships between the state variables.

Based on the paper's aim and scope, the authors must apply some simplifying assumptions corresponding with the problem under investigation. The most relevant simplification is connected with the paper character. The aim of the study is to state a coupled thermomechanical model as previously mentioned. In order to achieve a model coupling, maximum simplifications of the particular phenomena are assumed.

Due to the aforementioned objectives, the following assumptions were adopted:

- the Otto cycle as a description of the engine working principle,

- a nonlinear kinematics of the crank mechanism,

- a dynamic value of crankshaft angle caused by the equilibrium of acting and resistance moments,

- numerical simulations based on the derived nonlinear governing equations,

- linear elasticity of the crank system reduced to the piston rod.

Based on the paper's introduction and objectives, it was stated that the realisation of the aim will need the following steps:

- modelling of a crank mechanism based on the selected assumptions,

- numerical simulations devoted to sensitivity analysis,

- the model identification based on the experimental data,

- the model aided investigation for recognition of the crucial discrepancies with currently utilised models.

Proper modelling of the mechanism and numerical simulations seems to be crucial for the presented work. The proposed assumptions make it possible to achieve preliminary results. 


\section{Crank mechanism model}

Determination of dynamic loads of the considered model of crank mechanism and hence the other basic engine parts, primarily a block and a cylinder head, are related to the determination of the kinematics of this system.

The diagram of the crank mechanism model is shown in figure 1, which also represents the following engine parts: O - crankshaft, A - crank pin, I - crank moment of inertia, B - piston pin, $\mathrm{M}$ - piston mass. Additionally, the following symbols describing the model configuration were introduced: $r$ - the crank length (distance between the crankshaft and crank pin axes), $l_{0}$ the connecting rod length (distance between the crank pin and piston pin axes), $\varphi$ - crank rotation angle. The piston displacement and velocity for any crank rotation angle $\varphi$ can be determined on the basis of mechanism configuration presented in figure 1. Determination formulas given for the piston displacement and velocity are presented respectively in equation (1) and (2).

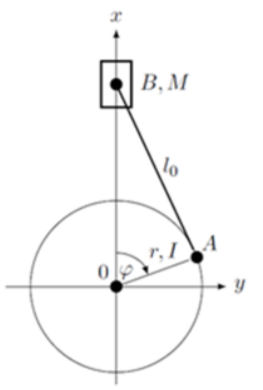

Figure 1. The scheme of the crank mechanism model Short description of figure.

$$
\begin{gathered}
x=r \cos (\varphi)+\sqrt{-r^{2} \sin ^{2}(\varphi)+\left(l_{o}+u\right)^{2}} \\
\dot{x}=-r \sin (\varphi) \dot{\varphi}+\frac{-r^{2} \sin (\varphi) \cos (\varphi) \dot{\varphi}+\left(l_{o}+u\right) \dot{u}}{\sqrt{-r^{2} \sin ^{2}(\varphi)+\left(l_{o}+u\right)^{2}}}
\end{gathered}
$$

Kinematic analysis of mechanisms is used to study motion and is an integral element of investigating the causes of existing motions that dynamics deals with. The dynamic analysis takes into account external forces, inertia forces, gravity forces and the force moments acting on the elements of the mechanism.

The kinetic energy was determined as the sum of the energy of the system mass of connecting rod $l_{0}$ and inertia moment of the crank $r$. The potential energy in the discussed system results from the elastic properties of the connecting rod $l_{0}$.

Hence, the Lagrangian (3) represents the total energy of the system under consideration presented in figure 1. The equation describing $L$ is given in (3):

$$
L=\frac{1}{2} I \dot{\varphi}^{2}+\frac{1}{2} M \dot{x}^{2}-\frac{k\left(r \cos (\varphi)+\sqrt{-r^{2} \sin ^{2}(\varphi)+\left(l_{o}+u\right)^{2}}-l_{o}\right)^{2}}{2}
$$

Nonholonomic model forces acting on the particular crank are caused by the other parts of a crankshaft, the entire powertrain or gas forces, which are the reason for stable operation of the engine. Thermodynamic force is the result of phenomenon connected with the particular stroke and instantaneous volume of the cylinder during operation. Depending on the assumed cycle, there is another thermodynamic transformation for 
the particular working stage. It states the relationship between the current value of the piston volume and the working pressure. After complex rearrangement of the formulas it is possible to determine an equation which relates the piston motion and the system pressure.

The considered engine load - Otto cycle is presented in figure 2 and has the following form:

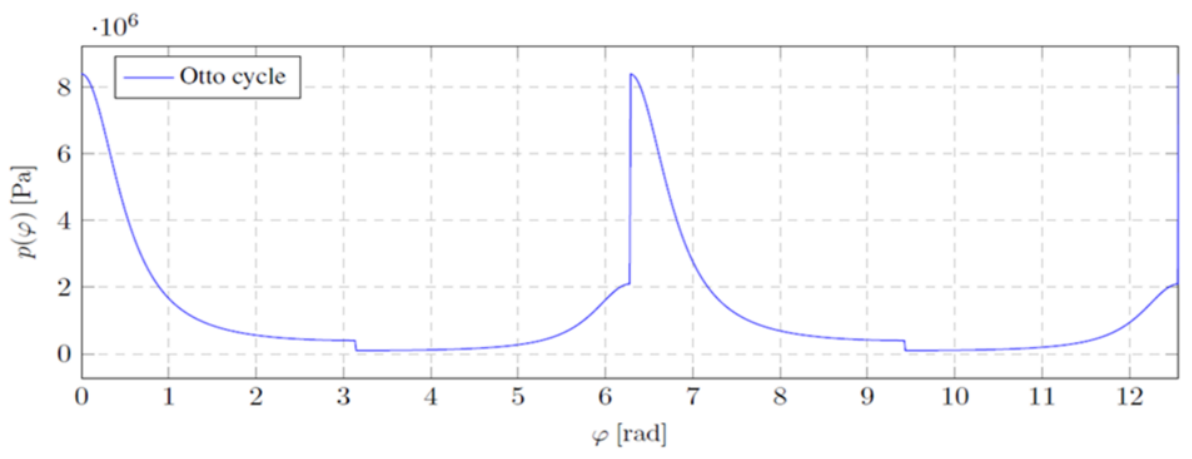

Figure 2. Otto cycle.

The assumed engine cycle describes particular stages of the engine operation. It assumes an infinite fast combustion and a process of temperature increasing. Moreover, it was adopted that compression and working cycle do not consider heat transfer (governed by an adiabatic transformation). Intake and exhaust strokes are given by an isobaric transformation with the atmospheric pressure.

\section{Description of the engine under investigation}

The considerations concern the TwinAir engine, which is a straight-twin type of an engine designed by Fiat Powertrain Technologies. The engine has hydraulically actuated variable valve timing and lift technology. It is offered in naturally aspirated and turbocharged variants. The TwinAir is popular for its reduced size, weight, fuel consumption and $\mathrm{CO}_{2}$ emissions.

The considerations concern the Fiat TwinAir engine since the author participated in the industrial research projects aimed at the process of redesigning a diesel engine vibration absorber [4], [5]. The engine body, head and crankshaft were provided in order to examine torsional vibration dampers on a built test stand. Having the engine geometrical parameters and mechanical properties enabled numerical analyses simulating real processes of combustion considering thermodynamic parameters of the Fiat TwinAir combustion chamber. On this basis the engine load was obtained by numerical computations.

The engine is characterized by the geometrical parameters and mechanical properties presented respectively in table 1 and 2 . 
Table 1. Fiat TwinAir - geometrical parameters.

\begin{tabular}{cc}
\hline \multicolumn{2}{c}{ TwinAir engine geometrical parameters } \\
\hline Geometrical parameter & Naturally aspirated \\
\hline Displacement & $1.01\left(964 \mathrm{~cm}^{3}\right)$ \\
Cylinder bore & $83.5 \mathrm{~mm}(3.29 \mathrm{in})$ \\
Piston stroke & $88 \mathrm{~mm}(3.5 \mathrm{in})$ \\
\hline
\end{tabular}

Table 2. Fiat TwinAir - mechanical properties.

\begin{tabular}{cc}
\hline \multicolumn{2}{c}{ TwinAir engine mechanical properties } \\
\hline Mechanical properties & Naturally aspirated \\
\hline Power output & $60 \mathrm{PS}(44 \mathrm{~kW})$ \\
Torque output & $88 \mathrm{Nm}$ at $3500 \mathrm{~min}^{-1}$ \\
Compression ratio & $11.2: 1$ \\
\hline
\end{tabular}

The information in tables 1 and 2 show that the object under investigation is a small engine with high operational properties. It indicates different dynamics of the device than for the older industrial solutions. Practical observations present the demand of the additional equipment to control the object dynamics and the most efficient tools for the design calculations. That causes a need for more detailed modelling of the engine, especially in the field of a transdisciplinary engineering.

\section{Numerical simulations}

In order to study the proposed dynamic coupled model, the authors performed some numerical simulations allowing for reliable evaluation of the modelling based on a transdisciplinary approach.

The plot depicted in figure 3 presents a crank displacement being influenced by the system's flexible properties. The linear curve was introduced for comparative purposes and shows the dynamic response of the uncoupled system where its rigidity was not included in the considerations. It can be stated that despite the small impact on dynamics in quantitative terms, qualitative changes in the dynamic response of the system can be captured. By analysing the oscillatory nature of the crank displacement, it can be concluded that the system equivalent stiffness is affected not only by the elastic properties of the gas force, having relatively less impact on the system dynamics, but mostly by the crank system stiffness taken into consideration. This indicates the importance of considerations of mutual relations between the thermodynamic and mechanical system properties as the latter are often omitted in the engine modelling process.

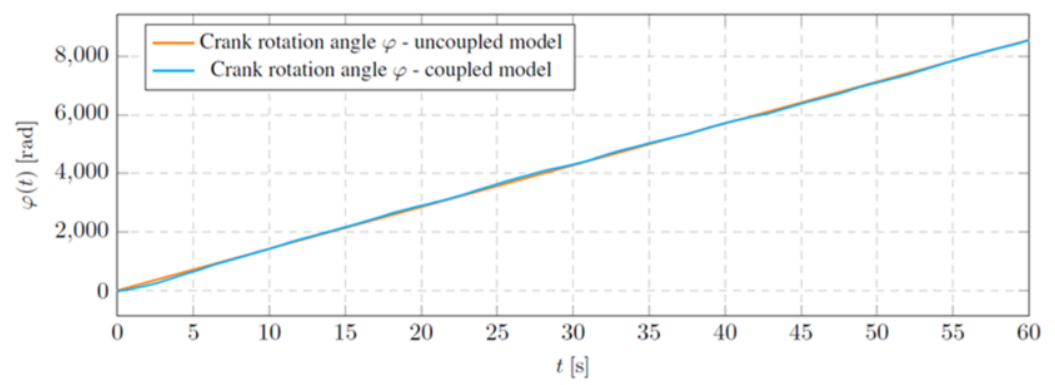

Figure 3. Crank rotation angle. 
More significant qualitative and quantitative changes can be seen on the crank rotational speed plot presented in figure 4 . The rotational speed oscillation is stable but not periodic. The results of simulations reveal a stochastic character of the engine dynamics. The phenomenon is compatible with empirical observations, which are uncorrelated and have random properties.

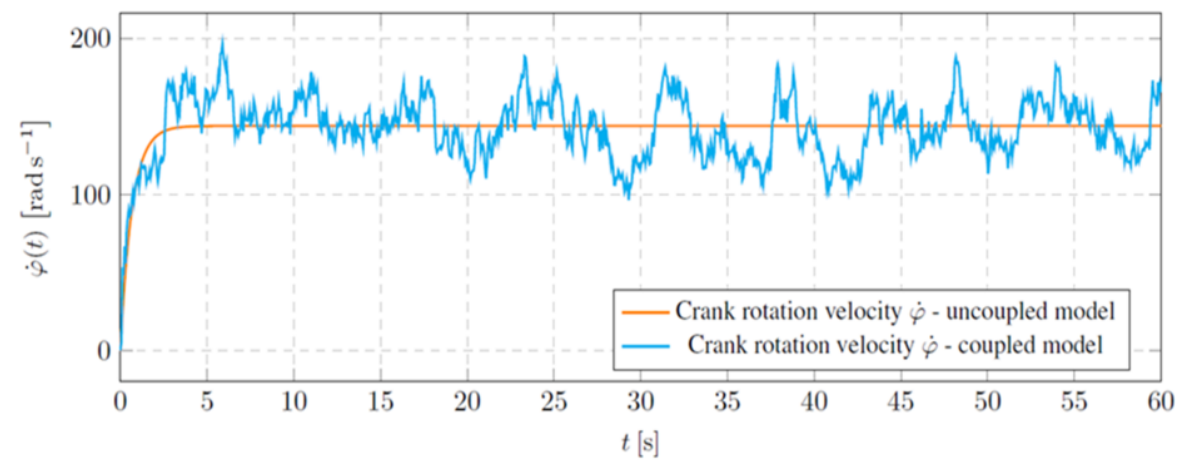

Figure 4. Crank angular velocity.

It can be stated that the obtained numerical simulations depict significant difference between an uncoupled mechanical approach and transdisciplinary modelling. The evaluated values have similar integral properties as a mean value or an average slope and very different dynamic features as a signal velocity or Root Mean Square (RMS) value.

Figure 5 depicts plots of gas forces considered in a modelling process of the engine dynamics. Gas forces computed based on the coupled (thermodynamic) and uncoupled (kinematic) model are presented and discussed as they reveal strong qualitative differences resulting in a different system dynamic response. The blue waveform represents a case of a kinematic-based engine load (due to displacements based on the uncoupled model) while the orange one stands for a fully dynamic gas force implementation (computed with the coupled model). Based on a comparative analysis, a repetitive, cyclic load in case of kinematically simulated gas force can be easily observed, while the changes of a dynamic engine load is a unique process related to the combustion process of the air-fuel mixture, which is never repeatable.

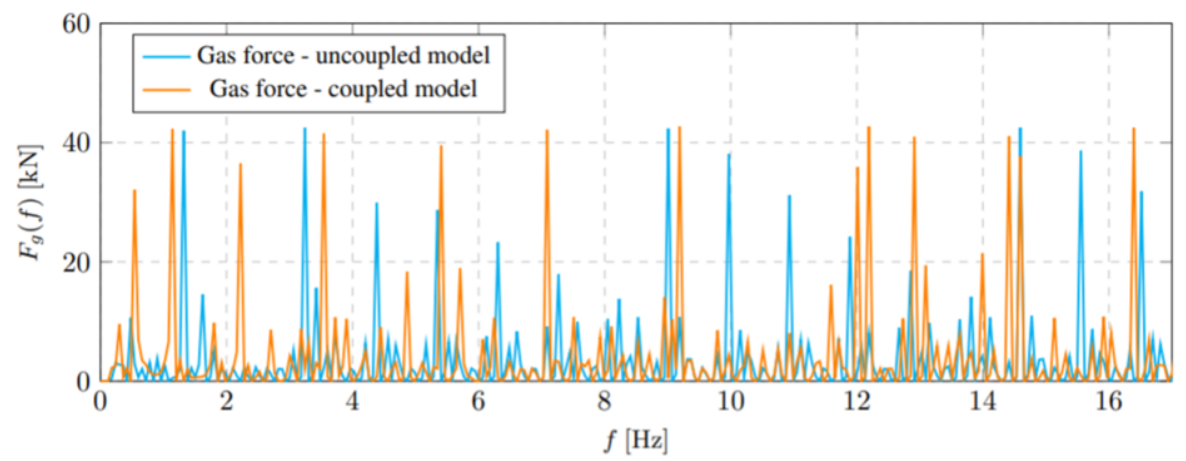

Figure 5. Gas force - comparative results. 
Non-periodical characters of transdisciplinary modelling results indicate the essential difference between the two types of numerical models' simulations. That leads to conclude the proposed model better represents an irregular character engine operation. Moreover, it can be stated that a random character of the subsequent working cycles can also be caused by the irregularity of the system velocity, not only by a stochastic process of combustion.

\section{Conclusions}

It can be concluded that the application of transdisciplinary engineering enables qualitatively new results which differ significantly from the results of a typical kinematic analysis of a combustion process in a reciprocating engine. On one hand, simulations which were carried out have similar general properties to the simpler models, especially in the area of the integral properties as a mean value or an average power. On the other hand, they are characterised by very different system dynamics, which reflects in a relatively high irregularity of the instantaneous value of the angular velocity. Comparatively to commonly utilised models, the performed analysis of the proposed uncoupled model delivers results which are closer to real engine dynamic behaviour.

The industrial research project creates a need of simple computational model of the engine for design processes of the Torsional Vibration Damper being Dynamic Vibration Absorber. Considering an operational principle of this kind of damper, the designed device has to be tuned to the power unit properties. Despite the model of simple double degree of freedom rotor was enough for this purpose, a determination of the excitation (caused by thermodynamic cycle) had to be supported by more sophisticated model. Articles and reports regarding the investigation present efficiency of sophisticated models in case of solving industrial problems [6, 14]. Transdisciplinary modelling of the engine operation enables to obtain relevantly better results as shown in the paper. Such an approach is necessary in order to select proper parameters of the designed device especially in case of the simultaneous fulfilling operational (expected engine dynamics) and functional conditions (desired inertial and geometrical parameters).

The carried out investigation presented in the subsequent sections of the paper allows for the formulation of the following conclusions:

1. As modern spark ignition engines become lighter, more efficient and more loaded than commonly used units, it results in different dynamics of the entire powertrain and also creates the demand for a more accurate model for the purposes of engine design, equipment selection and powertrain control. The transdisciplinary engineering approach enables the preparation of a tool that meets the above requirements.

2. The model proposed by the authors takes into account more details of crank system stiffness, gas force elasticity, viscous and inertial properties and enables the investigation of their mutual relationships. It results in a complex but solvable problem. The obtained result expands on models known in literature but keeps all the properties of simpler models. 
3. The results of the performed investigation present complex dynamics of the engine outputs as an angular displacement or velocity. It is strictly connected with nonlinear features of the proposed coupled model. The authors assumed that excitation in the form of a gas force generated in the combustion process depends not only on time but also on state variables. It results in a nonlinear system with extremely different dynamic properties. From the vibrational point of view, it is a selfexcited system characterised by the limit cycle which is the same operational velocity of the engine.

The proposed approach can be used to offer results qualitatively compatible with existing engines but there are some areas where even better results can be obtained. Some weakness of the presented method is the simplified model of the gas force. This will lead to some discrepancies when comparing to experimental data but still external outputs of the engine model are close to reality which brings the conclusion that even the proposed simplified methodology can be used successfully.

This transdisciplinary modelling approach might contribute in process of engines design being upgraded in terms of technical level what constitutes the added value resulting from a merge of two disciplines thermodynamics and mechanics of machines.

\section{References}

[1] B. Bellakhdhar, A. Dogui and J.-L. Ligier, A simplified coupled crankshaft-engine block model, Comptes Rendus Mecanique, 2013, Vol. 341, pp. 743-754.

[2] E. Brusa, C. Delprete and G. Genta, Torsional vibration of crankshafts: effects of non-constant moments of inertia, Journal of sound and vibration, 1997, Vol. 205, pp. 135-150.

[3] J.V. Chelsom, Concurrent engineering case studies: Lessons from Ford Motor Company Experience. In: C.S. Syan, U. Menon (eds.) Concurrent Engineering. Springer, Dordrecht, 1994, pp. 25-48.

[4] Grant No. NN5095 74639 - Modelling and design of the torsional vibration dampers of drive systems (2012-2015).

[5] Chilinski, B. Grant No. PBS1/B6/11/2012 - High-strength torsional vibration dampers of the crankshafts (2012-2015).

[6] B. Chilinski and M. Zawisza, Analysis of bending and angular vibration of the crankshaft with a torsional vibrations damper. Journal of Vibroengineering, 2016, 18, 5353-5363.

[7] M. Desbazeille, R. Randall, F. Guillet, M. El Badaoui and C. Hoisnard, Model-based diagnosis of large diesel engines based on angular speed variations of the crankshaft, Mechanical Systems and Signal Processing, 2010, Vol. 24, pp. 1529-1541.

[8] G. Iosif, I. Iordache, V. Stoica, A.M. Luchian, E. Costea, G. Suciu and V. Suciu, Achieving a More Electric Aircraft: a comparative study between the concurrent and traditional engineering models, INCAS Bulletin, 2018, Vol. 10, pp. 221-228.

[9] H. Karabulut, Dynamic model of a two-cylinder four-stroke internal combustion engine and vibration treatment, International Journal of Engine Research, 2012, Vol. 13, pp. 616-627.

[10] S. Lattanzio, E. Carey, A. Hultin, R. Imani Asrai, M. McManus, N. Mogles, G. Parry and L.B. Newnes, Transdisciplinarity within the Academic Engineering Literature, International Journal of Agile Systems and Management, 2020, Vol. 13, No. 2, pp. 213-232.

[11] J. Pokojski, Integration of Knowledge Based Approach and Multi-Criteria Optimization in MultiDisciplinary Machine Design, Advances in Transdisciplinary Engineering, Vol. 2, 2015, pp. 683 692.

[12] Q.H. Qin and C.X. Mao, Coupled torsional-flexural vibration of shaft systems in mechanical engineering-I. Finite element model, Computers \& Structures, 1996, Vol. 58, pp. 835-843.

[13] J.K. Sinha, F. Gu, L. Lidstone and A.D. Ball, Detecting the crankshaft torsional vibration of diesel engines for combustion related diagnosis, Journal of Sound and Vibration, 2009, Vol. 321, pp. 1171-1185. 
[14] B. C. Zbigniew Dabrowski, Identification of a model of the crankshaft with a damper of torsional vibrations. Journal of Sound and Vibration, 2017, Vol. 19, pp. 539-548. 